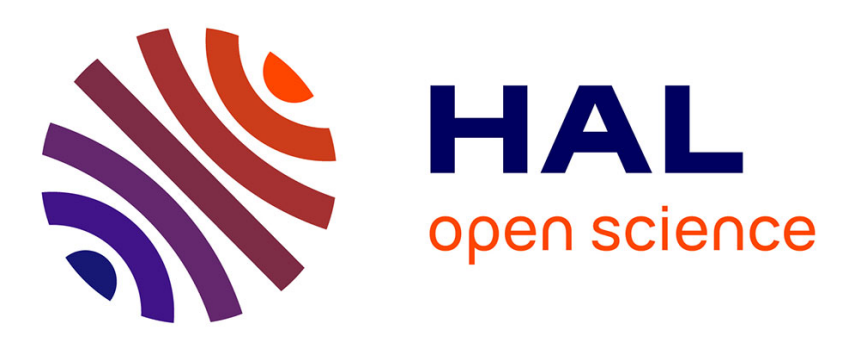

\title{
Guest editors' introduction to the special issue on Model Driven Engineering and Reverse Engineering: Research and Practice
}

Francesca Arcelli Fontana, Hugo Bruneliere, Hausi A. Müller, Claudia Raibulet

\section{To cite this version:}

Francesca Arcelli Fontana, Hugo Bruneliere, Hausi A. Müller, Claudia Raibulet. Guest editors' introduction to the special issue on Model Driven Engineering and Reverse Engineering: Research and Practice. Journal of Systems and Software, 2020, 159, pp.110446. 10.1016/j.jss.2019.110446 . hal02339020

\section{HAL Id: hal-02339020 \\ https://hal.science/hal-02339020}

Submitted on 9 Jan 2020

HAL is a multi-disciplinary open access archive for the deposit and dissemination of scientific research documents, whether they are published or not. The documents may come from teaching and research institutions in France or abroad, or from public or private research centers.
L'archive ouverte pluridisciplinaire HAL, est destinée au dépôt et à la diffusion de documents scientifiques de niveau recherche, publiés ou non, émanant des établissements d'enseignement et de recherche français ou étrangers, des laboratoires publics ou privés. 


\section{Guest editors' introduction to the special issue on Model Driven Engineering and Reverse Engineering: Research and Practice}

The systematic use of models in software engineering represents the foundation of Model Driven Engineering (or MDE). High-level domain-specific models are defined and further exploited for the implementation, testing, integration, and maintenance of software. One of the key ideas in MDE is that transformation of models from a high abstraction level to a lower level can be described and automated by using transformation languages. Source code can be then generated by limiting the amount of hand written code, implying less effort and possibly less errors.

Moreover, during its life cycle, existing software needs maintenance, modification and evolution independently of its size and application domain. Most of the times, the related documentation (e.g., concerning its architecture, features, knowledge) quickly becomes obsolete. Hence, most companies are facing the problem of performing reverse engineering tasks to comprehend the software to be changed. In this context, models can play a key role for improving software understanding processes, by providing different views on existing software at a higher abstraction level than source code. Hence, Model Driven Reverse Engineering (or MDRE) approaches start from the source code, at a low abstraction level, and produce different kinds of models of the software at higher abstraction levels. These models represent various perspectives on the same software. Depending on the cases, model discovery from source code and further model transformations can be more or less automated. As a result, the obtained models can be analyzed by domain experts and tools, or used as inputs to model-based processes (e.g., for forward engineering activities).

This special issue contains papers reporting on various research efforts, experiences or practices in the area of Model Driven Engineering (MDE) and Model Driven Reverse Engineering (MDRE). These papers are the result of a rigorous peer-reviewing and selection process that started in March 2018 (the submission deadline was actually extended up to April 16, 2018 due to several requests). At this date, we got 13 full paper submissions in total. Among them, and after one-to-three rounds of reviews by at least 2 reviewers for each paper, 6 full papers were finally selected for publication in the present special issue.

The papers contained in this volume cover a variety of MDE/MDRE-related topics going from extensions of the Knowledge Discovery Metamodel for aspect-orientation, metamodels for software protection and reverse engineering, structured assurance metamodels to model-driven IT governance process for long term objectives, retrieving behavioral model from black-box system, to approaches for reverse engineering of legacy systems and code migration. We propose an overview for each one of them in what follows.

The paper entitled "Evaluating the Extension Mechanisms of the Knowledge Discovery Metamodel for Aspect-Oriented Modernizations" by Santos et al., presents extensions of the Knowledge-Discovery Metamodel (KDM) to support aspect-orientation. The authors propose a 
heavyweight and a lightweight extension mechanisms based on the two extensions ways provided by KDM: the extensions of the existing metaclasses and the use of stereotypes. One of the main objectives of this paper is to study the two extensions from the suitability point of view (e.g., time needed for the software engineers to use the extension, errors made by software engineers in the extension process). This comparative study based on an actual experiment has pointed out the advantages and disadvantages of the two extensions outlining the main differences between them.

The paper entitled "A Meta-Model for Software Protections and Reverse Engineering Attacks" by Basile et al., defines a first metamodel which structures a knowledge base of software protection and reverse engineering attacks against man-in-the-loop attacks. Reverse engineering attacks aim to steal confidential assets from commercial code and to break the software integrity in unauthorized ways. To allow both humans and tools to reason about the strength of available combinations of protections against potential reverse engineering attacks, all available knowledge on the relations between the relevant aspects of protections, attacks, applications, and assets need to be collected, structured, and formalized. Basile et al., presents a software protection metamodel that can be instantiated to construct a formal knowledge base that holds such information. In addition, the authors validated their approach against available models in the software protection domain and developed prototype tools for non-modelling-expert software defenders to help them creating the knowledge base. All the tools are available in open source and have been applied to open source applications and industrial case studies.

The paper entitled "Model Based System Assurance Using the Structured Assurance Case Metamodel" by Wei et al., describes the Structured Assurance Case Metamodel 2.0 (SACM), a meta-model developed by the Object Management Group (OMG) and discuss how to use this metamodel to create a complete assurance case. Assurance cases are used to demonstrate confidence in system properties such as safety and security, and they are usually applied in safety-critical domains. The authors provide SACM compliant metamodels for existing system assurance approaches such as Goal Structuring Notation (GSN) and Claims, Arguments and Evidence (CAE). The authors also developed tools support exploiting the Eclipse environment to translate existing (e.g., graphical) systems assurance approaches to model-based system assurance using SACM.

The paper entitled "A Model-Driven IT Governance Process based on the Strategic Impact Evaluation of Services" by Wautelet describes a model-driven IT governance framework through which it is possible to study the alignment of the IT services to long-term objectives such as strategic and acquisition objectives. Such objectives lead an organization to enhance its competitive position in time. The framework addresses the three stages of the IT governance: evaluate, direct, and monitor. It supports supervision of the alignment with the to-be situation for pipeline services and with the as-is situation for catalog services. The framework has been validated through an actual case study, i.e., a Belgian hospital which activities and services have known a significant growth in the last years. 
The paper entitled "hW-inference: a Heuristic Approach to Retrieve Models through Black Box Testing" by Groz et al., proposes an approach to retrieve behavioral models from black-box (i.e., no source or binary code is needed) reactive software systems. The approach does not require resetting the system during the model inference. The input of the approach is represented by its input space, no further knowledge of a system is required. The output of the approach is expressed in terms of a Finite State Machine (FSM). The authors underline the scalability of their approach up to systems that may reach thousands of state. The authors have validated their approach on a typical control software system (e.g., a heating management system).

The paper entitled "Developing a Model-Driven Reengineering Approach for Migrating PL/SQL Triggers to Java: A Practical Experience" by Candel et al., proposes a process for reengineering projects based on model-driven principles. The authors describe well-defined processes to develop, test, and validate different transformations in a model-driven approach. The reengineering process integrates a test-driven development approach which develops incrementally model transformations with three kinds of validations for the generated code. Furthermore, they study the suitability of the different models used in the reengineering process and identify measurements of the effort needed for the transformation tasks. Candel et al. implemented also a code migration tool from Oracle Forms written using PL/SQL code to the Java platform. This tool achieves nearly a $100 \%$ rate of conversion. The paper presents also a practical experience, starting from a legacy code which is further described in terms of Knowledge Discovery Metamodel (KDM) models.

Many individuals contributed to the success of this special issue. Firstly, we would like to thank all the authors who submitted their work (whether it has been finally published or not). Secondly, we would like to thank all the reviewers who provided timely and high-quality reviews at the different steps of the revision process. Finally, we acknowledge the help and gracious support of Gemma Upton (Journal Manager of the Journal of Systems and Software) and Chan Wing Kwong (Editor of the Journal of Systems and Software). We really hope that the readers enjoy the papers from this special issue!

Francesca Arcelli Fontana, Universita' degli Studi di Milano-Bicocca, Italy Hugo Bruneliere, IMT Atlantique \& LS2N (CNRS), Nantes, France Hausi Müller, University of Victoria, Canada Claudia Raibulet, Universita' degli Studi di Milano-Bicocca, Italy 